MATEC Web of Conferences 1, 05003 (2012)

DOI: $10.1051 /$ matecconf $/ 20120105003$

(C) Owned by the authors, published by EDP Sciences, 2012

\title{
Aeroelastic instability analysis of NES-controlled systems via a mixed Multiple Scale/Harmonic Balance algorithm
}

\author{
Angelo Luongo $^{1, \mathrm{a}}$ and Daniele Zulli ${ }^{1}$ \\ DISAT - University of L'Aquila, Via Salaria Antica Ovest, 67100 L'Aquila (AQ) - Italy
}

\begin{abstract}
The issue to passively control aeroelastic instability of general nonlinear multi-d.o.f. systems, suffering Hopf bifurcation, is addressed. The passive device consists of an essentially nonlinear oscillator (Nonlinear Energy Sink), having the task to transfer energy from the main to the secondary structure. The mathematical problem is attacked by a new algorithm, based on a suitable combination of the Multiple Scale and the Harmonic Balance Methods. The procedure is able to furnish the codimension-2 invariant manifold on which the motion occurs, thus revealing the passive character of the oscillations of the NES. The algorithm also provides the bifurcation equations, which govern the slow flow on the manifold, expressed in terms of the main structure amplitude and phase of motion. It is shown that NES, under suitable conditions, can shift forward the bifurcation point, and, moreover, it can reduce the amplitude of the limit cycles. Theory is applied to a sample structure already studied in literature, consisting of a two-d.o.f. rigid wing under steady wind. Relevant asymptotic results are compared, for validation purposes, with numerical simulations.
\end{abstract}

\section{Introduction}

Nonlinear Energy Sinks (NES) are constituted by essentially nonlinear oscillators, attached to a main structure. Their mass is generally small, compared to that of the main structure, and their goal is to absorb energy and to dissipate it, as a passive control device, through targeted energy transfers (TET) (see [1,2] for an extensive background). Their main feature, consequence of the essential nonlinear nature, consists in the capacity of oscillate at any frequencies, giving rise of large band tuning with the structure to be controlled. Recently, these kind of devices have received great attention in the literature, being used in many applications. In [3,4], multiple parallel NESs have been considered to dissipate first-mode oscillations of a linear structure under impulse as well as harmonic forcing. In [5] non-smooth NES has been considered to control a twod.o.f. system. In [6], NES is used to suppress aeroelastic instabilities on a rigid wing, modeled as a two-d.o.f. sectionmodel, under stationary wind. To study the slow-flow dynamics, the authors make use of the complexification procedure by Manevitch [7] and, then, the Multiple Scale Method [8].

In this paper, inspired by [6], a general, nonlinear, multid.o.f. system, close to a Hopf bifurcation point, is considered, and a NES is attached to it in order to control amplitude of vibrations. A mixed Multiple Scale/Harmonic Balance Method (MSHBM) is applied to get the invariant manifold and the equations ruling the slow-dynamics on it. The main advantage of the algorithm is that the initial complexification procedure is avoided and it is re-conducted in

\footnotetext{
a e-mail: angelo.luongo@univaq.it
}

the framework of the classical perturbation techniques. A sample system, already analyzed in [6], is then considered, for which the asymptotic results are compared with numerical integrations.

The paper is organized as follows: in Section 2, the algorithm is applied to a general system, with or without NES; in Section 3 the sample system is studied, and in Section 4 some conclusions are drawn.

\section{The Multiple Scale/Harmonic Balance algorithm}

A quite general class of nonlinear, multi-d.o.f. mechanical systems, close to a Hopf bifurcation, caused by aerodynamic forces, is considered (see Fig. 1). The main system is equipped with an essentially nonlinear oscillator behaving as a nonlinear energy sink (NES), attached at a point. The relevant nondimensional equations of motion reads:

$$
\begin{gathered}
\mathbf{M} \ddot{\mathbf{x}}+\mathbf{C}(\mu) \dot{\mathbf{x}}+\mathbf{K}(\mu) \mathbf{x}+\xi\left(\mathbf{r}^{T} \dot{\mathbf{x}}-\dot{y}\right) \mathbf{r} \\
+\kappa\left(\mathbf{r}^{T} \mathbf{x}-y\right)^{3} \mathbf{r}+\mathbf{f}(\mathbf{x}, \mathbf{x}, \mathbf{x})=0 \\
m \ddot{y}-\xi\left(\mathbf{r}^{T} \dot{\mathbf{x}}-\dot{y}\right)-\kappa\left(\mathbf{r}^{T} \mathbf{x}-y\right)^{3}=0
\end{gathered}
$$

where: $\mathbf{x}=\mathbf{x}(t)$ is the time-depending $N$-dimensional vector of the displacements of the main structure; $\mathbf{M}$ is the mass matrix; $\mathbf{C}(\boldsymbol{\mu})$ is the (non-proportional) damping matrix and $\mathbf{K}(\mu)$ is the stiffness matrix, both including structural and aerodynamic effects, the latter depending on the nondimensional wind velocity $\mu$, acting as bifurcation parameter; $\mathbf{f}$ is the vector of the (cubic) geometric nonlinearities, assumed much larger than the (neglected) aerody-

This is an Open Access article distributed under the terms of the Creative Commons Attribution License 2.0, which permits unrestricted use, distribution, and reproduction in any medium, provided the original work is properly cited. 


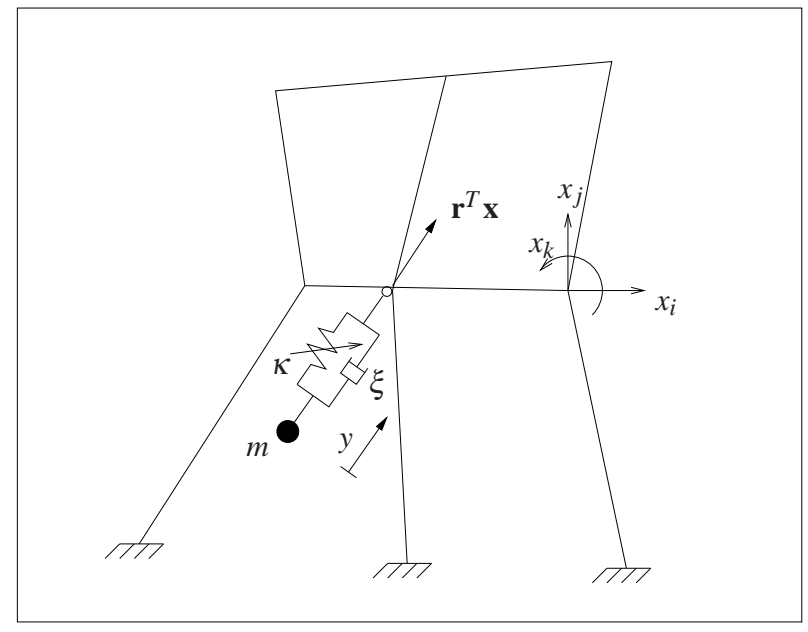

Figure 1. Sketch of a multi-d.o.f. system equipped with a NES.

namic nonlinearities, $y=y(t)$ is the time-depending displacement of the added oscillator, $m$ its (small) mass, $\xi$ its (small) damping-ratio and $\kappa$ the coefficient of its essentially nonlinear (cubic) spring; $\mathbf{r}$ is the influence coefficient vector; finally, the dot represents time-differentiation. It is convenient to introduce the relative displacement between main structure and NES, $z:=\mathbf{r}^{T} \mathbf{x}-y$, so that the equations (1) and (2) become:

$$
\begin{aligned}
& \mathbf{M} \ddot{\mathbf{x}}+\mathbf{C}(\mu) \dot{\mathbf{x}}+\mathbf{K}(\mu) \mathbf{x}+\xi \dot{z} \mathbf{r}+\kappa z^{3} \mathbf{r}+\mathbf{f}(\mathbf{x}, \mathbf{x}, \mathbf{x})=0 \\
& m\left(\mathbf{r}^{T} \ddot{\mathbf{x}}-\ddot{z}\right)-\xi \dot{z}-\kappa z^{3}=0
\end{aligned}
$$

It is interesting to note that, when $\xi=0$, the tangent operator of Eqs. (3) and (4) at $\mathbf{x}=\mathbf{0}$, admits a double-zero eigenvalue, due to the fact the NES has zero linear stiffness. The unfolding of this system is a family in which a small damping $\xi$ and a small linear stiffness $\kappa_{L}$ exist. Moreover, if the main structure manifests a Hopf bifurcation at $\mu=\mu_{0}$, a double-zero-Hopf bifurcation would occur at the point $\left(\mu_{0}, 0,0\right)$ of the parameter space $\left(\mu, \xi, \kappa_{L}\right)$, similarly to what studied in [9]. According to the Center Manifold Theory [10], such a circumstance would lead to the conclusion that, when $t \rightarrow \infty$, the motion develops itself on a four-dimensional manifold. However, such a bifurcation is not a standard one, since the mass of NES is evanescent, so that, in the limit for $m \rightarrow 0$, the two eigenvalues of NES assume undetermined values. Such a pathological case, therefore, cannot be studied by the usual Center Manifold Method, or by the Multiple Scale Method, but a specific perturbation method must be tailored on it.

To this end, the dependent variables are first rescaled through a nondimensional small parameter $\varepsilon>0$, as $(\mathbf{x}, z):=$ $\varepsilon^{1 / 2}(\tilde{\mathbf{x}}, \tilde{z})$, consistently with the presence of cubic nonlinearity, while the bifurcation parameter is expressed as $\mu=$ $\mu_{0}+\varepsilon \mu_{1}$, where $\mu_{0}$ is its critical value, to be still evaluated, and $\varepsilon \mu_{1}$ is the small deviation from it. The parameters of the NES are also rescaled, since both the mass and the damping are assumed small: $(m, \xi):=\varepsilon(\tilde{m}, \tilde{\xi})$. The rescaling leads to the following equations, after omission of tilde and division by $\varepsilon^{1 / 2}$ :

$$
\begin{aligned}
& \mathbf{M} \ddot{\mathbf{x}}+\left(\mathbf{C}_{0}+\varepsilon \mu_{1} \mathbf{C}_{1}\right) \dot{\mathbf{x}}+\left(\mathbf{K}_{0}+\varepsilon \mu_{1} \mathbf{K}_{1}\right) \mathbf{x}+\varepsilon \xi \dot{z} \mathbf{r}+ \\
& \quad+\varepsilon \kappa z^{3} \mathbf{r}+\varepsilon \mathbf{f}(\mathbf{x}, \mathbf{x}, \mathbf{x})=0 \\
& \varepsilon m\left(\mathbf{r}^{T} \ddot{\mathbf{x}}-\ddot{z}\right)-\varepsilon \xi \dot{z}-\varepsilon \kappa z^{3}=0
\end{aligned}
$$

where $\mathbf{C}_{0}:=\mathbf{C}\left(\mu_{0}\right), \mathbf{K}_{0}:=\mathbf{K}\left(\mu_{0}\right)$ and $\mathbf{C}_{1}:=\partial \mathbf{C}\left(\mu_{0}\right) / \partial \mu, \mathbf{K}_{1}:=$ $\partial \mathbf{K}\left(\mu_{0}\right) / \partial \mu$.

According to the the Multiple Scale Method, independent time scales $t_{0}:=t, t_{1}:=\varepsilon t, \ldots$ are introduced and, consistently, the derivatives expressed as $\frac{d}{d t}=d_{0}+\varepsilon d_{1}+$ $\ldots$ and $\frac{d^{2}}{d t^{2}}=d_{0}^{2}+2 \varepsilon d_{0} d_{1}+\ldots$. Moreover, the dependent variables are expanded in series as:

$$
\left\{\begin{array}{l}
\mathbf{x} \\
z
\end{array}\right\}=\left\{\begin{array}{l}
\mathbf{x}_{0} \\
z_{0}
\end{array}\right\}+\varepsilon\left\{\begin{array}{l}
\mathbf{x}_{1} \\
z_{1}
\end{array}\right\}+\ldots
$$

Substituting in Eqs. (5) and (6) and collecting terms of the same order in $\varepsilon$, leads to the following perturbation equations:

order $\varepsilon^{0}$ :

$$
\mathbf{M} d_{0}^{2} \mathbf{x}_{0}+\mathbf{C}_{0} d_{0} \mathbf{x}_{0}+\mathbf{K}_{0} \mathbf{x}_{0}=0
$$

order $\varepsilon^{1}$ :

$$
\begin{aligned}
& \mathbf{M} d_{0}^{2} \mathbf{x}_{1}+\mathbf{C}_{0} d_{0} \mathbf{x}_{1}+\mathbf{K}_{0} \mathbf{x}_{1}=-2 \mathbf{M} d_{0} d_{1} \mathbf{x}_{0}-\mathbf{C}_{0} d_{1} \mathbf{x}_{0} \\
& \quad-\mu_{1} \mathbf{C}_{1} d_{0} \mathbf{x}_{0}-\mu_{1} \mathbf{K}_{1} \mathbf{x}_{0}-\xi d_{0} z_{0} \mathbf{r} \\
& \quad-\kappa z_{0}^{3} \mathbf{r}-\mathbf{f}\left(\mathbf{x}_{0}, \mathbf{x}_{0}, \mathbf{x}_{0}\right) \\
& m\left(\mathbf{r}^{T} \ddot{\mathbf{x}}_{0}-\ddot{z}_{0}\right)-\xi \dot{z}_{0}-\kappa z_{0}^{3}=0
\end{aligned}
$$

It should be noticed, that, because of the vanishingly small values of the mass and damping, as well of the lack of linear stiffness, the equation of motion relevant to NES does not appear in the generator problem Eq. (8), which therefore describes the linear dynamics of the main structure only. It is assumed that, at the specific critical value $\mu_{0}$, the system experiences a Hopf bifurcation. This entails that the relevant eigenvalue problems

$$
\begin{array}{r}
\left(\lambda^{2} \mathbf{M}+\lambda \mathbf{C}_{0}+\mathbf{K}_{0}\right) \mathbf{u}=\mathbf{0} \\
\left(\bar{\lambda}^{2} \mathbf{M}^{T}+\bar{\lambda} \mathbf{C}_{0}^{T}+\mathbf{K}_{0}^{T}\right) \mathbf{v}=\mathbf{0}
\end{array}
$$

have a solution $\lambda_{1,2}= \pm i \omega$, with the associated right ( $\mathbf{u}$ and $\overline{\mathbf{u}}$ ) and left ( $\mathbf{v}$ and $\overline{\mathbf{v}}$ ) eigenvectors (the overbar denoting the complex conjugate and $i$ the imaginary unit), while all the other eigenvalues have negative real parts and are far from the imaginary axis. Under these hypotheses, the steady solution of Eq. (8) is:

$$
\mathbf{x}_{0}\left(t_{0}, t_{1}, \ldots\right)=A\left(t_{1}, \ldots\right) \mathbf{u} e^{i \omega t_{0}}+c c
$$

where $A\left(t_{1}, \ldots\right)$ is a complex amplitude whose modulation on the slower time-scales must be evaluated, and $c c$ stands for complex conjugate.

The $\varepsilon$-order perturbation equations (9) and (10) are now addressed, and the NES equation considered first. Since 


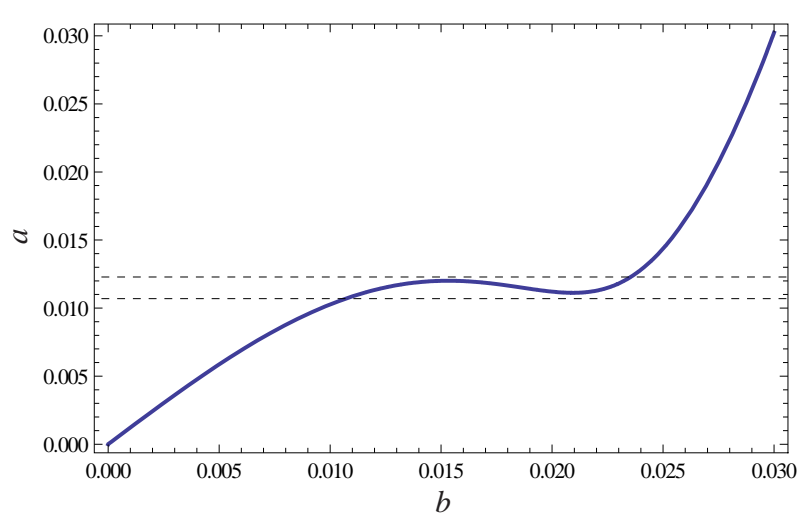

Figure 2. Constrain provided by the NES for the specific numeric example.

its (steady) solution cannot be expressed by elementary (nor Jacobi) functions, the Harmonic Balance Method is used, by letting:

$$
z_{0}\left(t_{0}, t_{1}, \ldots\right)=\sum_{k} B_{k}\left(t_{1}, \ldots\right) e^{i k \omega t_{0}}+c c
$$

where $B_{k}\left(t_{1}, \ldots\right)$ are slowly modulated complex amplitudes to be evaluated. Equations (13) and (14) are substituted in Eq. (10) and $\omega$ frequency terms only balanced. This step, after coming back to the true time, leads to

$$
-m \omega^{2}\left(B-\mathbf{r}^{T} \mathbf{u} A\right)+i \xi \omega B+3 \kappa B^{2} \bar{B}=0
$$

where $B:=B_{1}$.

Equation (15) provides an algebraic constrain between the (active) amplitude of oscillation of the main structure and the (passive) amplitude of the NES elongation, $B$; it therefore describes a codimension-2 manifold in the statespace, on which the asymptotic dynamics takes place.

To get the polar form of Eq. (15), $A(t):=\frac{1}{2} a(t) e^{i \alpha(t)}$ and $B(t):=\frac{1}{2} b(t) e^{i \beta(t)}$, are substituted in it and real and imaginary parts separated, thus obtaining:

$$
\begin{gathered}
m \omega^{2}\left(b-\mathbf{r}^{T} \mathbf{u} a \cos \gamma\right)-\frac{3}{4} \kappa b^{3}=0 \\
-m \omega^{2} \mathbf{r}^{T} \mathbf{u} a \sin \gamma-\xi \omega b=0
\end{gathered}
$$

where $\gamma:=\alpha-\beta$ denotes the phase difference. Equations (16) and (17) can be manipulated to eliminate the phasedifference, to get

$$
\frac{9}{16} \frac{\kappa^{2}}{\omega^{2}} b^{6}-\frac{3}{2} \kappa m b^{4}+\left(\xi^{2}+m^{2} \omega^{2}\right) b^{2}-m^{2} \omega^{2}\left(\mathbf{r}^{T} \mathbf{u}\right)^{2} a^{2}=0
$$

Equation (18) can be solved in the form $a=a(b)$, but usually it cannot be inverted in the form $b=b(a)$. For the specific sample system which will be detailed later, the manifold is shown in Fig. 2. It is evident how, for certain values of $a$ (in the range $(0.010,0.012)$, marked off by the dashed lines), the $b$-solution is multivalued.

Equation (9) is then addressed, in which $z_{0}$ is now known. By requiring that the resonant forcing term is orthogonal to the null space of the adjoint operator (solvability condition), the following condition is obtained:

$$
c_{1} \dot{A}=\mu_{1} c_{2} A+c_{3} B+c_{4} A^{2} \bar{A}+c_{5} B^{2} \bar{B}
$$

where the true time has been reintroduced, and the expression of the complex coefficients $c_{i}$ is given in Appendix A. It is worth noticing that, when $B=0$ is put into Eq. (19), this reduces to the normal form equation for the Hopf bifurcation of the main system. This entails that the NES modifies both the bifurcation point and the limit cycle, thus bringing potential benefits to the mechanical behavior of the original system. In polar coordinates, it becomes:

$$
\begin{gathered}
c_{1 R} \dot{a}-c_{1 I} a \dot{\alpha}=\mu_{1} c_{2 R} a+\left(c_{3 R} \cos \gamma+c_{3 I} \sin \gamma\right) b \\
+\frac{c_{4 R}}{4} a^{3}+\left(c_{5 R} \cos \gamma+c_{5 I} \sin \gamma\right) \frac{b^{3}}{4} \\
c_{1 I} \dot{a}+c_{1 R} a \dot{\alpha}=\mu_{1} c_{2 I} a+\left(c_{3 I} \cos \gamma-c_{3 R} \sin \gamma\right) b \\
+\frac{c_{4 I}}{4} a^{3}+\left(c_{5 I} \cos \gamma-c_{5 R} \sin \gamma\right) \frac{b^{3}}{4}
\end{gathered}
$$

where $c_{i R}$ and $c_{i I}$ are the real and imaginary parts of $c_{i}$, respectively. Equations (20) and (21) can be further combined to eliminate $\dot{\alpha}$, thus getting:

$$
\begin{aligned}
\eta_{11} \dot{a}= & \mu_{1} \eta_{12} a+\left(\eta_{13} \cos \gamma+\eta_{13}^{*} \sin \gamma\right) b \\
& +\eta_{14} \frac{a^{3}}{4}+\left(\eta_{15} \cos \gamma+\eta_{15}^{*} \sin \gamma\right) \frac{b^{3}}{4}
\end{aligned}
$$

which is in the form $\dot{a}=F(a, b, \gamma)$. The coefficients $\eta_{i j}$ and $\eta_{i j}^{*}$ are given in Appendix A. This differential equation (bifurcation equation), under the algebraic constraints (16),(17) (manifold equations) rules the evolution of the dynamics of the structure with NES.

\subsection{Periodic responses without NES}

When the NES is not attached to the main structure, the response is ruled just by Eq. (22), with $b \equiv 0$. The equilibrium points $\dot{a}=0$, correspond to periodic solutions for the original variables $\mathbf{x}$. In addition to the trivial solution $a_{e} \equiv 0$, a nontrivial solution is obtained:

$$
\begin{aligned}
& a_{e}=2 \sqrt{-\frac{\eta_{12}}{\eta_{14}} \mu_{1}} \\
& \dot{\alpha}_{e}=-\frac{\eta_{24}^{*}}{\eta_{14}} \mu_{1}
\end{aligned}
$$

The stability of the trivial solution will be discussed later, when NES is considered too. On the other hand, the stability of the nontrivial solution (23), (24) is addressed by substituting $a=a_{e}+\delta a, \dot{a}=\delta \dot{a}, b=0$ in Eq. (22), and retaining the linear terms in $\delta a$ only. It is well-known that these steps lead to the following outcome: the nontrivial branch is stable if super-critical, or unstable if sub-critical. 


\subsection{Periodic responses with NES}

If the NES is attached to the main system, the whole differentialalgebraic system, constituted by Eqs. (16),(17), (22), has to be solved. The trivial solution $a=0, b=0$, is still admitted by the system, while mono-modal solutions, in which just one of the two amplitudes is different from zero, do not exist, according to the passive character of $b$. To evaluate non-trivial solutions $a \neq 0, b \neq 0$, it is convenient to combine the equations, in order to eliminate the variables $a$ and $\gamma$. The procedure leads to (see Appendix B):

$$
\mathscr{F}(b) \dot{b}=\mathscr{G}(b)
$$

where $\mathscr{F}(b)$ and $\mathscr{G}(b)$ are polynomials in $b$. Once $b$ is obtained from this equation, $a$ and $\gamma$ are evaluated by the expressions in Appendix B.

Steady solutions $b_{e}$ of Eq. (25) are sought putting $\mathscr{G}(b)=$ 0 , and stability is ruled by the sign of $\frac{d}{d t}\left(\frac{\mathscr{G}\left(b_{e}\right)}{\mathscr{F}\left(b_{e}\right)}\right)$.

The stability of the trivial solution is now addressed. Substitution $A=\delta A, \dot{A}=\delta \dot{A}, B=\delta B$ in Eqs. (15) and (19), and retaining the linear terms in $\delta A, \delta B$, leads to

$$
\begin{aligned}
& c_{1} \delta \dot{A}=\mu_{1} c_{2} \delta A+c_{3} \delta B \\
& m \omega^{2}\left(\delta B-\mathbf{r}^{T} \mathbf{u} \delta A\right)+i \xi \omega \delta B=0
\end{aligned}
$$

Elimination of $\delta B$ provides:

$$
c_{1} \delta \dot{A}=\left(\mu_{1} c_{2}+c_{3} \frac{\mathbf{r}^{T} \mathbf{u}}{m \omega^{2}+i \xi \omega}\right) \delta A
$$

Here the term $c_{3} \frac{\mathbf{r}^{T} \mathbf{u}}{m \omega^{2}+i \xi \omega}$ represents the contribution of the NES to the stability of the trivial solution. Therefore, the NES modifies the position of the bifurcation point, i.e. the value of $\mu$ corresponding to the critical condition. Putting $\delta A:=p+i q$ and $\delta \dot{A}:=\dot{p}+i \dot{q}$ and separating real and imaginary parts one obtains

$$
\mathbf{L}\left\{\begin{array}{l}
\dot{p} \\
\dot{q}
\end{array}\right\}=\mathbf{J}\left\{\begin{array}{l}
p \\
q
\end{array}\right\}
$$

where

$$
\begin{aligned}
& \mathbf{L}=\left[\begin{array}{cc}
c_{1 R} & -c_{1 I} \\
c_{1 I} & c_{1 R}
\end{array}\right], \quad \mathbf{J}=\left[\begin{array}{ll}
J_{11} & J_{12} \\
J_{21} & J_{22}
\end{array}\right] \\
& J_{11}=-\mu_{1} c_{2 R}-\frac{m \omega \mathbf{r}^{T} \mathbf{u}}{\xi^{2}+m^{2} \omega^{2}}\left(c_{3 I} \xi-c_{3 R} m \omega\right) \\
& J_{12}=\mu_{1} c_{2 I}+\frac{m \omega \mathbf{r}^{T} \mathbf{u}}{\xi^{2}+m^{2} \omega^{2}}\left(c_{3 R} \xi+c_{3 I} m \omega\right) \\
& J_{21}=-J_{12} \\
& J_{22}=J_{11}
\end{aligned}
$$

The eigenvalues, not reported here, are the roots of the algebraic system $\operatorname{det}(\lambda \mathbf{L}-\mathbf{J})=0$; their real parts rule the stability of the trivial equilibrium.

\section{Sample system and numerical results}

A sample system, already studied in [6], is considered here to check the reliability of the method presented in Section 2. The main system represents a 2-d.o.f. section model of an airfoil under steady-state wind, capable of a cross-wind translation $x$ and a twist rotation $\varphi$, for which terms in Eq. (1) specialize as:

$$
\begin{aligned}
& \mathbf{x}=\left\{\begin{array}{l}
x \\
\varphi
\end{array}\right\}, \mathbf{M}=\left[\begin{array}{cc}
1 & n_{12} \\
n_{12} & n_{22}
\end{array}\right], \mathbf{C}=\mu\left[\begin{array}{ll}
g_{11} & 0 \\
g_{21} & 0
\end{array}\right], \\
& \mathbf{K}=\left[\begin{array}{cc}
\Omega^{2} & \mu^{2} g_{11} \\
k_{21} & n_{22}-\mu^{2} g_{21}
\end{array}\right], \mathbf{r}=\left\{\begin{array}{l}
1 \\
r
\end{array}\right\}, \mathbf{f}=\left\{\begin{array}{l}
f_{1} x^{3} \\
f_{2} \varphi^{3}
\end{array}\right\}
\end{aligned}
$$

and numerical values are: $n_{12}=n_{21}=0.2, n_{22}=0.25$, $g_{11}=0.2, g_{21}=-0.08, \Omega=0.5, k_{21}=0, r=0.9, f_{1}=$ $f_{2}=1, m=0.02, \xi=0.008, \kappa=40$.

The (nondimensional) critical wind velocity is found to be $\mu_{0}=0.87$, at which the system experiences a Hopf bifurcation. The associated frequency is $\omega=0.87$, the right eigenvector is $\mathbf{u}=\{0,1\}^{T}$ and the left eigenvector is $\mathbf{v}=$ $\{-0.241+0.191 i, 1\}^{T}$. The right eigenvector indicates that, at the leading order, only the rotation $\varphi$ is involved in the dynamics, while the translation $x$ is a higher-order variable.

When a NES is attached to the airfoil, it oscillates at an amplitude $b$, which is related to the amplitude $a$ of the eigenvector $\mathbf{u}$, as already shown in Fig. 2. The steady solutions of the airfoil, without and with NES, are shown in Fig. 3 vs. the wind velocity $\mu$. The red line represents solutions obtained without NES: at $\mu=\mu_{0}$ the trivial solution loses stability and a Hopf bifurcation occurs, corresponding to the emanation of a stable bifurcated branch. The effect of the NES (blue line) is to shift the bifurcation to higher value of the wind velocity ( $\mu \simeq 0.92$ ), where a low-amplitude, stable, bifurcated branch emanates from. For increasing wind, this branch experiences a loose of stability at $\mu=1$ and a subsequent fold at $\mu \simeq 1.12$. The unstable branch exists up to $\mu \simeq 0.82$, where a second turning point occurs. Here, the branch re-gains stability and causes higher amplitudes than those without NES, in coexistence with the lower-amplitude solutions. They are reached for different initial conditions (making this system unsuitable to NES). The results are confirmed by good agreement with numerical integrations of the equations of motion (1)(2), represented by colored points.

In Figure 4, the corresponding plots of $b$ and $\dot{\alpha}$ are shown vs. $\mu$.

\section{Conclusions}

In this paper, a general, nonlinear, multi-d.o.f. system, close to a Hopf bifurcation point, equipped with an essentially nonlinear oscillator with small mass (Nonlinear Energy Sink, NES), is considered. Aim of the NES is to passively control the amplitude of vibrations of the main system. A mixed 

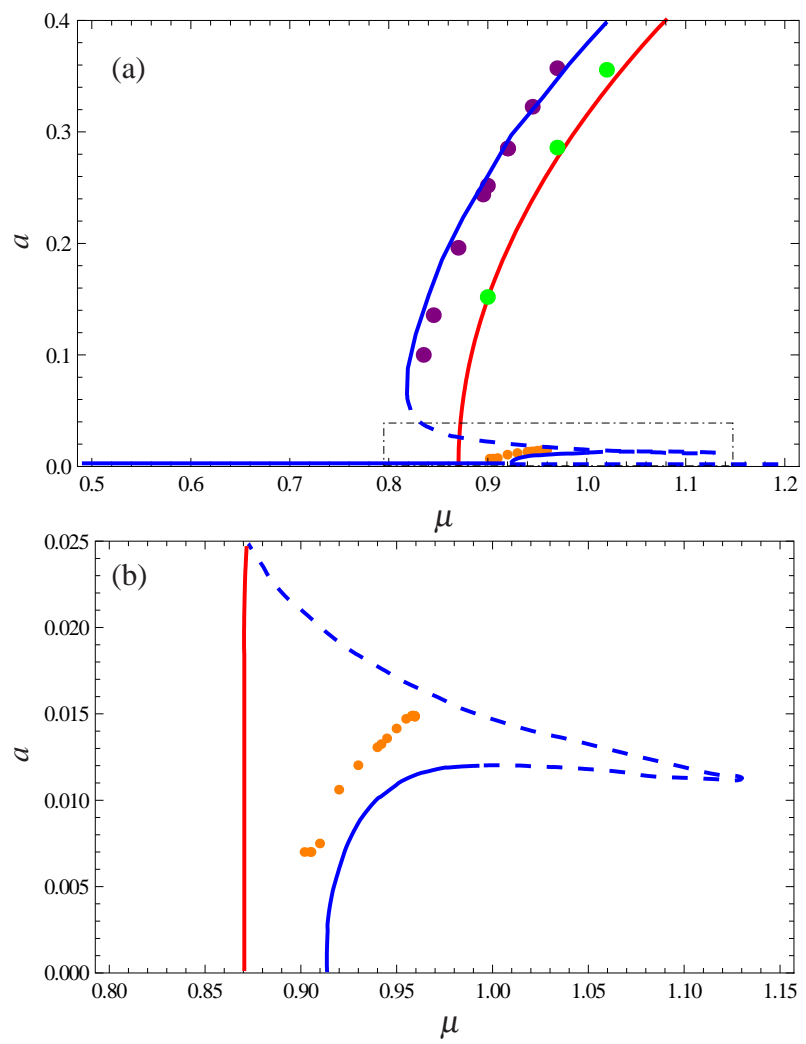

Figure 3. Amplitude $a$ vs. $\mu$, without NES (red line) and with NES (blue line). Plot (b) is a zoom of plot (a) in the dash-dot region. Continuous: stable; dashed: unstable; points: numerical integrations.

Multiple Scale/Harmonic Balance Method is proposed to get slow-flow equations, as well as the invariant manifold on which the dynamics occurs. This latter works a constrain between the amplitude of oscillations of the main structure and NES. The main advantage of the procedure is that no complexification is required, so that the analysis is re-conducted in the framework of the perturbation techniques. Numerical tests on a specific example, already analyzed in [6], constituted by a two-d.o.f. rigid wing under subsonic wind, are pursued. The results show good agreement between the outcomes of the algorithm and direct integrations. The presence of NES modifies the position of the bifurcation point and the amplitude of the bifurcated branch. However, as also recognized in [6], the presence of NES in this specific system is not always beneficial, since high amplitude oscillations coexist with regimes of controlled small-amplitude oscillations.

\section{A Coefficients of the equations}

The expression of the coefficients of Eq. (19) are:

$$
\begin{aligned}
& c_{1}=-2 i \omega \mathbf{v}^{H} \mathbf{M u}-2 \mathbf{v}^{H} \mathbf{C}_{0} \mathbf{u} \\
& c_{2}=i \omega \mathbf{v}^{H} \mathbf{C}_{1} \mathbf{u}+\mathbf{v}^{H} \mathbf{K}_{1} \mathbf{u} \\
& c_{3}=i \xi \omega \mathbf{v}^{H} \mathbf{r}
\end{aligned}
$$
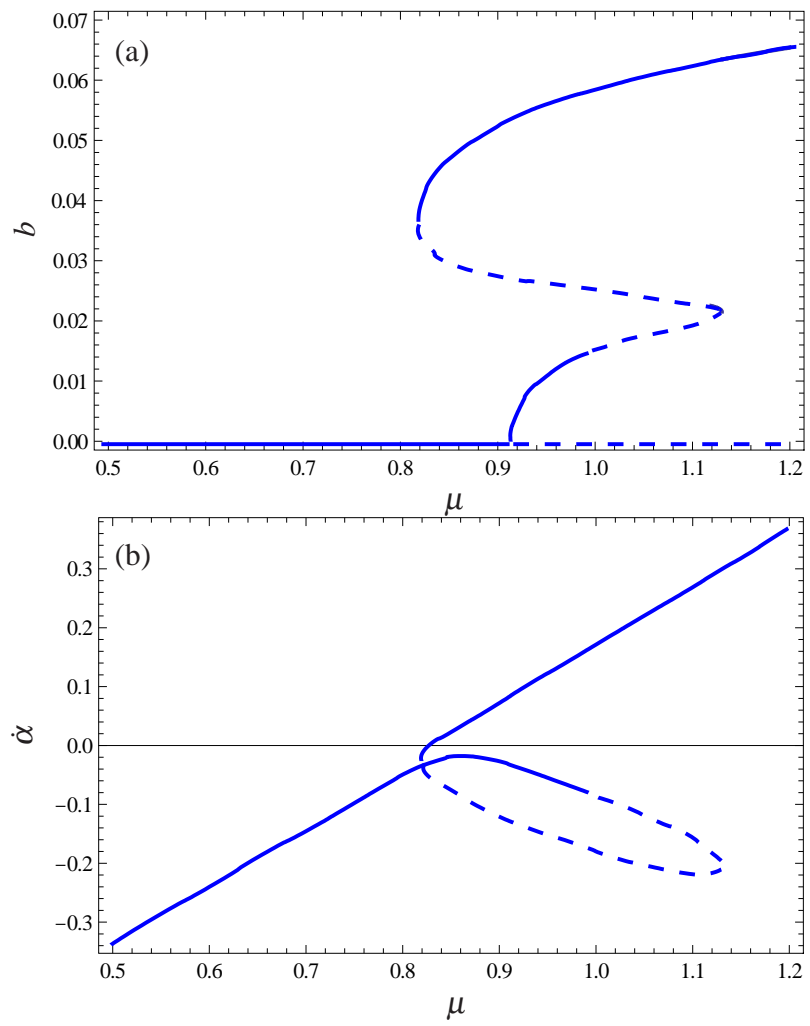

Figure 4. Values of $b$ (plot (a)) and $\dot{\alpha}($ plot (b)) and vs. $\mu$. Continuous: stable; dashed: unstable.

$$
\begin{aligned}
& c_{4}=3 \mathbf{v}^{H} \mathbf{f}(\mathbf{u}, \mathbf{u}, \mathbf{u}) \\
& c_{5}=3 \kappa \mathbf{v}^{H} \mathbf{r}
\end{aligned}
$$

where $H$ stands for Hermitian (transpose conjugate).

About the coefficients of Eq. (22), defining $\mathbf{c}_{i}:=\left\{c_{i R}, c_{i I}\right\}^{T}$, then $\eta_{i j}:=\mathbf{c}_{i}^{T} \mathbf{c}_{j}$ and $\eta_{i j}^{*}:=\left\|\mathbf{c}_{i} \times \mathbf{c}_{j}\right\|$, where $\times$ is the vector product.

\section{B Manipulation of the system with NES}

Some manipulations can be performed to get a simpler form of the differential-algebraic system (16), (17), (20), (21), when $a \neq 0$ and $b \neq 0$ : (a) both the sides of Eqs. (16) and (17) are multiplied by $b$ and then solved to get expressions for $a b \cos \gamma$ and $a b \sin \gamma$, just in terms of even powers of $b$ :

$$
\begin{aligned}
& a b \cos \gamma=\mathscr{F}_{1}\left(b, b^{3}\right) \\
& a b \sin \gamma=\mathscr{F}_{2}(b)
\end{aligned}
$$

where $\mathscr{F}_{i}$ are linear operators; (b) from Eq. (18), an expression for $a^{2}$, in terms of even powers of $b$, is obtained: $a^{2}=\mathscr{F}_{3}\left(b^{2}, b^{4}, b^{6}\right)$; (c) both the members of Eqs (20) and (21) are multiplied by $a$ :

$$
\begin{gathered}
c_{1 R} a \dot{a}-c_{1 I} a^{2} \dot{\alpha}=\mathscr{F}_{4}\left(a^{2}, a^{4}, a b \cos \gamma, a b \sin \gamma,\right. \\
\left.a b^{3} \cos \gamma, a b^{3} \sin \gamma\right) \\
c_{1 I} a \dot{a}+c_{1 R} a^{2} \dot{\alpha}=\mathscr{F}_{5}\left(a^{2}, a b \cos \gamma, a b \sin \gamma,\right. \\
\left.a b^{3} \cos \gamma, a b^{3} \sin \gamma\right)
\end{gathered}
$$




\section{MATEC Web of Conferences}

(d) expressions obtained in points (a) and (b) are substituted in Eqs. (43) and (44), and $a \dot{a}$ is substituted by $2 \frac{d a^{2}}{d t}$; (e) expression for $\frac{d a^{2}}{d t}$ is obtained from differentiation of what obtained in point (b): $\frac{d a^{2}}{d t}=\frac{d \mathscr{F}_{3}}{d b} \dot{b}$, and substituted in Eqs. (43) and (44); (f) the second equation is multiplied by $\frac{c_{1 I}}{c_{1 R}}$ and summed to the first.

At the end of this procedure, a scalar nonlinear differential equation, depending only on $b$, is obtained (Eq. (25)) where the expressions of $\mathscr{F}(b)$ and $\mathscr{G}(b)$ are:

$$
\begin{gathered}
\mathscr{F}(b)=\frac{b^{5}}{\left(\mathbf{r}^{T} \mathbf{u}\right)^{2}}\left(\frac{27 c_{1 I} \kappa^{2}}{16 c_{1 R} m^{2} \omega^{4}}+\frac{27 c_{1 R} \kappa^{2}}{16 m^{2} \omega^{4}}\right) \\
+\frac{b^{3}}{\left(\mathbf{r}^{T} \mathbf{u}\right)^{2}}\left(-\frac{3 c_{1 I}{ }^{2} \kappa}{c_{1 R} m \omega^{2}}-\frac{3 c_{1 R} \kappa}{m \omega^{2}}\right) \\
+\frac{b}{\left(\mathbf{r}^{T} \mathbf{u}\right)^{2}}\left(\frac{c_{1 I}}{c_{1 R}}+\frac{c_{1 I} \xi^{2}}{c_{1 R} m^{2} \omega^{2}}+c_{1 R}+\frac{c_{1 R} \xi^{2}}{m^{2} \omega^{2}}\right)
\end{gathered}
$$

and

$$
\mathscr{G}(b)=h_{1} b^{2}+h_{2} b^{4}+h_{3} b^{6}+h_{4} b^{8}+h_{5} b^{10}+h_{6} b^{12}(46)
$$

where

$$
\begin{aligned}
& h_{1}=\frac{c_{1 I} c_{2 I}}{c_{1 R}\left(\mathbf{r}^{T} \mathbf{u}\right)^{2}}+\frac{c_{1 I} c_{2 I} \xi^{2}}{c_{1 R}\left(\mathbf{r}^{T} \mathbf{u}\right)^{2} m^{2} \omega^{2}}-\frac{c_{1 I} c_{3 R} \xi}{c_{1 R}\left(-\mathbf{r}^{T} \mathbf{u}\right) m \omega} \\
& -\frac{c_{1 I} c_{3 I}}{c_{1 R}\left(-\mathbf{r}^{T} \mathbf{u}\right)}+\frac{c_{2 R}}{\left(\mathbf{r}^{T} \mathbf{u}\right)^{2}}+\frac{c_{2 R} \xi^{2}}{\left(\mathbf{r}^{T} \mathbf{u}\right)^{2} m^{2} \omega^{2}}-\frac{c_{3 R}}{\left(-\mathbf{r}^{T} \mathbf{u}\right)} \\
& +\frac{c_{3 I} \xi}{\left(-\mathbf{r}^{T} \mathbf{u}\right) m \omega} \\
& h_{2}=-\frac{3 c_{1 I} c_{2 I} \kappa}{2 c_{1 R}\left(\mathbf{r}^{T} \mathbf{u}\right)^{2} m \omega^{2}}+\frac{3 c_{1 I} c_{3 I} \kappa}{4 c_{1 R}\left(-\mathbf{r}^{T} \mathbf{u}\right) m \omega^{2}} \\
& -\frac{c_{1 I} c_{5 R} \xi}{4 c_{1 R}\left(-\mathbf{r}^{T} \mathbf{u}\right) m \omega}-\frac{c_{1 I} c_{5 I}}{4 c_{1 R}\left(-\mathbf{r}^{T} \mathbf{u}\right)}-\frac{3 c_{2 R} \kappa}{2\left(\mathbf{r}^{T} \mathbf{u}\right)^{2} m \omega^{2}} \\
& +\frac{3 c_{3 R} \kappa}{4\left(\mathbf{r}^{T} \mathbf{u}\right) m \omega^{2}}-\frac{c_{5 R}}{4\left(-\mathbf{r}^{T} \mathbf{u}\right)}+\frac{c_{5 I} \xi}{4\left(-\mathbf{r}^{T} \mathbf{u}\right) m \omega} \\
& +\frac{c_{4 R}}{4\left(-\mathbf{r}^{T} \mathbf{u}\right)^{4}}+\frac{c_{4 R} \xi^{4}}{4\left(\mathbf{r}^{T} \mathbf{u}\right)^{4} m^{4} \omega^{4}}+\frac{c_{4 R} \xi^{2}}{2\left(\mathbf{r}^{T} \mathbf{u}\right)^{4} m^{2} \omega^{2}} \\
& h_{3}=\frac{9 c_{1 I} c_{2 I} \kappa^{2}}{16 c_{1 R}\left(\mathbf{r}^{T} \mathbf{u}\right)^{2} m^{2} \omega^{4}}+\frac{3 c_{1 I} c_{5 I} \kappa}{16 c_{1 R}\left(-\mathbf{r}^{T} \mathbf{u}\right) m \omega^{2}} \\
& +\frac{9 c_{2 R} \kappa^{2}}{16\left(\mathbf{r}^{T} \mathbf{u}\right)^{2} m^{2} \omega^{4}}+\frac{3 c_{5 R} \kappa}{16\left(-\mathbf{r}^{T} \mathbf{u}\right) m \omega^{2}} \\
& -\frac{3 c_{4 R} \kappa \xi^{2}}{4\left(\mathbf{r}^{T} \mathbf{u}\right)^{4} m^{3} \omega^{4}}-\frac{3 c_{4 R} \kappa}{4\left(\mathbf{r}^{T} \mathbf{u}\right)^{4} m \omega^{2}} \\
& h_{4}=\frac{9 c_{4 R} \kappa^{2} \xi^{2}}{32\left(\mathbf{r}^{T} \mathbf{u}\right)^{4} m^{4} \omega^{6}}+\frac{27 c_{4 R} \kappa^{2}}{32\left(\mathbf{r}^{T} \mathbf{u}\right)^{4} m^{2} \omega^{4}} \\
& h_{5}=\frac{27 c_{4 R} \kappa^{3}}{64\left(\mathbf{r}^{T} \mathbf{u}\right)^{4} m^{3} \omega^{6}} \\
& h_{6}=\frac{81 c_{4 R} \kappa^{4}}{1024\left(\mathbf{r}^{T} \mathbf{u}\right)^{4} m^{4} \omega^{8}}
\end{aligned}
$$

\section{References}

1. A.F. Vakakis, O.V. Gendelman, L.A. Bergman, D.M. McFarland, G. Kerschen, Y.S. Lee, Nonlinear Targeted Energy Transfer in Mechanical and Structural Systems, I (Springer-Verlag, New York, 2008)

2. A.F. Vakakis, O.V. Gendelman, L.A. Bergman, D.M. McFarland, G. Kerschen, Y.S. Lee, Nonlinear Targeted Energy Transfer in Mechanical and Structural Systems, II (Springer-Verlag, New York, 2008)

3. B. Vaurigaud, A.T. Savadkoohi, C.-H. Lamarque, "Targeted energy transfer with parallel nonlinear energy sinks. Part I: Design theory and numerical results", Nonlinear Dyn., DOI 10.1007/s11071-011-9949-x (2011)

4. A.T. Savadkoohi, B. Vaurigaud, C.-H. Lamarque, S. Pernot, "Targeted energy transfer with parallel nonlinear energy sinks. Part II: Design theory and experiments", Nonlinear Dyn., DOI 10.1007/s11071-0119955-z (2011)

5. C.-H. Lamarque, O.V. Gendelman, A.T. Savadkoohi, E. Etcheverria, "Targeted energy transfer in mechanical systems by means of non-smooth nonlinear energy sink", Acta Mech., DOI 10.1007/s00707-011-0492-0 (2011)

6. O.V. Gendelman, A.F. Vakakis, L.A. Bergman, D.M. McFarland, "Asymptotic Analysis of Passive Nonlinear Suppression of Aeroelastic Instabilities of a Rigid Wing in Subsonic Flow", SIAM J. Appl. Math., 70, n. 5 (2010), 1655-1677

7. L. Manevitch, "The Description of Localized Normal Modes in a Chain of Nonlinear Coupled Oscillators Using Complex Variables", Nonlinear Dyn., 25 (2001), 95109

8. A.H. Nayfeh, D.T. Mook, Nonlinear oscillations. (John Wiley and Sons, New York, 1979)

9. A. Luongo, D. Zulli, "A Multiple Scale Algorithm for Double-Zero/Hopf Bifurcation”, Nonlinear Dyn., submitted (2011)

10. J. Guckenheimer and P. Holmes. Nonlinear Oscillations, Dynamical Systems, and Bifurcation of Vector Fields, (Springer-Verlag, New York, 1983). 